\title{
Obstáculos para el aprendizaje del modelo de evolución por selección natural, en estudiantes de escuela secundaria de Argentina*
}

\section{Obstacles for the learning of the model of evolution by natural selection in students of secondary school in Argentina}

\author{
Leonardo González Galli ${ }^{1}$ Elsa Meinardi ${ }^{1}$
}

\begin{abstract}
Resumen: En este trabajo se presentan los resultados de una investigación tendiente a identificar los principales obstáculos para el aprendizaje del modelo de evolución por selección natural en estudiantes de dos cursos de secundaria. La existencia de dichos obstáculos se infiere a partir de la identificación y caracterización de los patrones explicativos y las concepciones que los estudiantes ponen en juego al explicar casos de evolución adaptativa, así como de análisis provenientes de la historia y epistemología de la biología y de la psicología cognitiva. Se identifican la teleología de sentido común, el razonamiento causal lineal y el razonamiento centrado en el individuo como tres obstáculos principales y se discuten algunas de las implicancias didácticas de estos resultados.
\end{abstract}

Palabras clave: Enseñanza de la biología. Evolución. Selección natural. Aprendizaje. Metacognición.

\begin{abstract}
In this work we present the results of research which intended to identify the principal obstacles for the learning of the model of evolution by natural selection in students belonging to two courses in secondary school. The existence of obstacles is deduced from the identification and characterization of the explicative patterns and the conceptions that the students express when they explain cases of adaptive evolution. This is supported by the analysis coming from the history and epistemology of biology and from cognitive psychology. It identifies common-sense teleology, linear causal reasoning and individual based reasoning as three main obstacles and some of the instructional consequences of those results are discussed.
\end{abstract}

Keywords: Biology teaching. Evolution. Natural selection. Learning. Metacognition.

\footnotetext{
*Este trabajo ha contado con financiamiento de la Universidad de Buenos Aires (proyecto UBACyT 1141) y del Consejo Nacional de Investigaciones Científicas y Técnicas (CONICET)

${ }^{1}$ Instituto de Investigaciones en Enseñanza de las Ciencias, Facultad de Ciencias Exactas y Naturales, Universidad de Buenos Aires, Boulogne Sur Mer, San Isidro, Buenos Aires, Argentina. E-mail: <leomgalli@gmail.com>
} 


\section{Introducción y referentes teóricos}

En este trabajo presentamos una parte de los resultados de la investigación de tesis doctoral del primer autor sobre los obstáculos para el aprendizaje del modelo de evolución por selección natural (MESN). El tema central de dicha investigación son los problemas asociados con la enseñanza y con el aprendizaje de los modelos de la biología evolutiva. Más específicamente, se aborda la cuestión de las denominadas "concepciones alternativas". En relación con este problema, se han producido numerosas investigaciones de carácter más bien descriptivo; en este trabajo, por el contrario, exploramos la naturaleza y funcionamiento de estas concepciones analizando los posibles supuestos generales subyacentes (obstáculos) que guían su funcionamiento. Asumimos que la identificación de estos obstáculos puede constituir un valioso insumo para el diseño de estrategias didácticas que favorezcan la construcción, por parte de los estudiantes, de modelos teóricos sobre la evolución más cercanos a los científicos y para el desarrollo de las capacidades metacognitivas.

\section{Dificultades en relación con la enseñanza y el aprendizaje de la teoría de la evolución}

La investigación en didáctica de las ciencias ha revelado que en numerosos países los estudiantes egresados de la enseñanza media, y el público general, demuestran una pobre comprensión de los principales conceptos de la biología evolutiva (ALTERS; NELSON, 2002; SMITH, 2010b). Entre los factores que dificultan la enseñanza y el aprendizaje de la teoría evolutiva se han identificado los siguientes:

- La presencia e influencia de valores e ideas religiosas tanto en estudiantes como en profesores (GRIFFITH; BREM, 2004; SMITH, 2010b).

- La presencia y persistencia de concepciones alternativas, no necesariamente asociadas al pensamiento religioso (BISHOP; ANDERSON, 1990; SMITH, 2010a).

- La inadecuación de los materiales y estrategias didácticos (DEMASTES; SETTLAGE JR.; GOOD, 1995; MARTINS; BRAGA, 2002; NEHM; SCHONFELD, 2007).

- El insuficiente desarrollo cognitivo de los estudiantes (KEOWN, 1988; LAWSON; THOMPSON, 1988).

- El escaso conocimiento y/o la no aceptación de la teoría de la evolución por parte de los profesores de ciencias (BERKMAN; PACHECO; LUTZER, 2008; SMITH, 2010b).

Este trabajo se relaciona con uno de estos factores: las concepciones alternativas de los estudiantes.

\section{Concepciones de los estudiantes en relación con la evolución adaptativa}

Son especialmente numerosos los estudios de tipo descriptivo producidos en el ámbito de la didáctica de las ciencias que muestran que estudiantes de todos los niveles educativos tienen concepciones sobre el proceso evolutivo que resultan claramente erróneas desde el punto de vista científico (por ejemplo, BISHOP; ANDERSON, 1990; JIMÉNEZ ALEIXANDRE, 1991; SETTTLAGE, 1994). Este resultado se ha repetido en numerosos países: España (JIMÉNEZ ALEIXANDRE, 2009), Israel (JUNGWIRTH, 1975), Inglaterra (BRUMBI, 1984), Australia 
(MARTIN, 1983), Suecia (HALLDEN, 1988), EE.UU (BISHOP; ANDERSON, 1990), Brasil, (MARTINS; BRAGA, 2002), México (MILLÁN BENÍTEZ; CARMONA PIÑA; ZÁRATE, 1997) y Argentina (MEINARDI; ADÚRIZ-BRAVO, 2002), entre otros. Esta conclusión puede ser extendida - en cierto grado - a los docentes encargados de enseñar estos temas (AFANATTO, 1986; ROSA et al., 2002; OSIF, 1997) y a muchos estudiantes y egresados de carreras universitarias relacionadas con la biología (ALTERS; NELSON, 2002; DAGHER; BOUJAUDE, 1997; MEINARDI; ADÚRIZ-BRAVO, 2002).

En relación con las concepciones alternativas numerosas investigaciones convergen en señalar que algunas de ellas parecen estar ampliamente difundidas y comparten los siguientes rasgos:

- Son, en ciertos aspectos, semejantes a la teoría lamarckiana de la evolución (JIMÉNEZ ALEIXANDRE, 1991).

- Suponen que el cambio evolutivo es consecuencia del cambio individual (BARDAPURKAR, 2008).

- Se basan en la noción de "necesidad" (SOUTHERLAND et al., 2001).

- Implican la noción de "uso y desuso" de estructuras orgánicas (PASSMORE; STEWART, 2002).

- Involucran la noción de "herencia de los caracteres adquiridos" (BANET; AYUSO, 2003).

- Suponen que el cambio evolutivo obedece a fines predeterminados (teleología) (KAMPOURAKIS; ZOGZA, 2008).

Muchos estudios destacan la naturaleza teleológica de las concepciones de los estudiantes. En relación con este último punto cabe destacar que dicho sesgo ha sido generalmente señalado como un rasgo puramente negativo de los razonamientos de los estudiantes. En contadas ocasiones se le ha reconocido cierto valor heurístico, por ejemplo, Zohar y Ginossar (1998); en este trabajo ofrecemos una visión alternativa de este problema.

\section{Explicaciones, concepciones y obstáculos para el aprendizaje}

Sugerimos que, a los fines de nuestro análisis, se puede considerar la existencia de una jerarquía cognitiva en términos de estructuras más generales que incluyen (y sesgan y constriñen) otras más específicas. Así, denominaremos "obstáculos" a los modos de razonar más generales e implícitos (ASTOLFI, 1994). En el otro extremo, denominaremos "explicaciones" o simplemente "respuestas" a los modos de razonar explícitos y más específicos evidenciados por los textos que los estudiantes producen para dar cuenta de un problema específico. En un nivel intermedio situamos las "concepciones". Dado el carácter implícito de los obstáculos, su existencia y características solo pueden ser inferidas indirectamente a partir de las explicaciones y

\footnotetext{
${ }^{2}$ Esta analogía entre las ideas de los estudiantes y la teoría de Lamarck está fuertemente cuestionada en la actualidad (GONZÁLEZ GALLI; MEINARDI, 2008; KAMPOURAKIS; ZOGZA, 2006).
} 
de las concepciones identificadas en dichas explicaciones. Por tal motivo, en nuestra investigación enfrentamos a los estudiantes con diversos problemas para los cuales produjeron explicaciones, a partir de las cuales identificamos las concepciones puestas en juego para, finalmente, inferir la existencia de posibles obstáculos.

Dado que el concepto de obstáculo es utilizado de diversas maneras en la literatura (ASTOLFI, 1994, 2001; CAMILLONI, 2001), proponemos una definición (GONZÁLEZ GALLI; MEINARDI, 2010) a la que nos remitiremos, según la cual se trata de una forma de pensar que cumple tres requisitos: (1) es transversal (se aplica a un campo amplio de contenidos); (2) es funcional (permite al sujeto explicar y explicarse ciertos fenómenos del mundo); y (3) compite con el modelo científico a aprender (ambos sistemas conceptuales dan cuenta de los mismos fenómenos).

\section{La teoría de la evolución}

Desde una perspectiva semántica de las teorías científicas consideramos que una teoría es un conjunto de modelos que pretende dar cuenta de ciertos aspectos del mundo (ADÚRIZ BRAVO, 2012). Esta forma de ver las teorías es especialmente adecuada en el caso de la "teoría de la evolución" ya que, en lugar de una teoría formal unificada, lo que encontramos son numerosos modelos, cada uno de los cuales pretende dar cuenta de algún aspecto particular del complejo proceso de cambio evolutivo (THOMPSON, 1989). Las dos principales cuestiones que dicha disciplina busca explicar son: el origen de la diversidad biológica y el de la adaptación (STERELNY; GRIFFITHS, 1999). Desde esta perspectiva, el MESN emerge como el principal (aunque no el único) modelo de la biología evolutiva, ya que constituye el principal recurso para explicar tanto el origen de nuevas especies como de la adaptación. De hecho, en relación con este último fenómeno, la mayoría de los expertos considera que el MESN constituye el único modelo capaz de explicar la evolución adaptativa (FUTUYMA, 2009; RIDLEY, 2004). Por estos motivos, y sin desconocer la existencia y relevancia de otros modelos (ver FOLGUERA; GONZÁLEZ GALLI, 2012; GONZÁLEZ GALLI; MEINARDI, 2013), enfocamos nuestra indagación en las dificultades asociadas con su aprendizaje.

\section{Objetivos de la investigación}

El objetivo principal de este trabajo fue identificar y caracterizar los principales patrones explicativos y las concepciones que los estudiantes ponen en juego al explicar casos de evolución adaptativa, para luego inferir los posibles obstáculos subyacentes. Sugerimos que este análisis puede suponer un aporte significativo a la mejora de la enseñanza de estos contenidos ya que tener en cuenta los obstáculos (más que cada concepción específica) al diseñar las estrategias didácticas puede resultar más fructífero, dado el carácter transversal de los mismos. 


\section{Metodología}

La población participante estuvo conformada por cincuenta y un estudiantes de dos cursos de cuarto año (dieciséis años de edad) de una escuela secundaria de la Ciudad Autónoma de Buenos Aires (Argentina).

El enfoque general de la investigación fue de tipo cualitativo-interpretativo (LATORRE BELTRÁN; RINCÓN IGEA; ARNAL, 1997). Los datos se obtuvieron mediante la aplicación de diversos instrumentos en dos instancias; una previa y otra posterior a la implementación de una unidad didáctica (UD). Las etapas del trabajo involucraron las siguientes instancias e instrumentos para la toma de datos (Cuadro 1).

- Instancia pre-instruccional (antes de la implementación de la UD)

- El "problema de los piojos" (PP) (un problema escrito de resolución abierta);

- Entrevistas semi-estructuradas individuales.

- Implementación de la UD (siete actividades).

- Instancia post-instruccional (después de la implementación de la UD)

- El "problema de los peces" (PF) (un problema escrito de resolución abierta aplicado inmediatamente después de la implementación de la UD);

- El "problema de los leones” (PL) (un problema escrito de resolución abierta aplicado un año después de la implementación de la UD).

El $\mathrm{PP}^{3}$ plantea a los estudiantes un caso de evolución adaptativa: la evolución de la resistencia a un insecticida en una población de piojos. Además se llevaron a cabo entrevistas semi-estructuradas individuales ${ }^{4}$ con todos los estudiantes participantes después de haber respondido por escrito el PP. Las entrevistas fueron grabadas y estuvieron orientadas a la ampliación de estas respuestas así como a la indagación de algunos tópicos específicos.

La UD estuvo centrada en la enseñanza del MESN y en la desestabilización del pensamiento finalista y consistió en siete actividades trabajadas durante diez clases. Esta UD parcialmente comunicada en González Galli, Revel Chion y Meinardi (2008) estuvo orientada a la "superación" del pensamiento teleológico mediante el desarrollo de una "vigilancia" consciente de naturaleza metacognitiva (PETERFALVI, 2001). El funcionamiento del pensamiento teleológico a modo de obstáculo se postuló como una hipótesis inicial de trabajo que se vio luego avalada por los resultados. Las respuestas escritas a todas las actividades de la UD también fueron fuente de datos para la identificación de concepciones y obstáculos. Las clases durante las cuales se implementó la UD estuvieron a cargo del investigador.

Inmediatamente después de finalizada dicha UD se aplicó el PF. La parte (a) del PF es equivalente, en cuanto a su estructura lógico-conceptual, al PP (se trata en ambos casos de explicar el origen de un rasgo adaptativo). La parte (b) del PF planteaba la pérdida de la visión en una población de peces cavernícolas, un caso de resolución más difícil para los estudiantes

\footnotetext{
${ }^{3}$ Se trata de una adaptación del problema propuesto por Jiménez Aleixandre (1991).

${ }^{4}$ Para el diseño e implementación de las entrevistas se siguieron los lineamientos generales sugeridos por Goetz y Lecompte (1988) y por Osborne y Freyberg (1995).
} 
(ESPINASA; ESPINASA, 2008). Finalmente, un año después de la implementación de la UD se aplicó (a los mismos estudiantes) el PL, el cual es conceptualmente equivalente al PP (y al punto [a] del PF). Así, las tres actividades (el PP, el punto (a) del PF y el PL) planteaban a los estudiantes la explicación de casos de evolución adaptativa conceptualmente equivalentes (origen de un rasgo adaptativo). Por el contrario, el punto (b) del PF planteaba un caso de explicación más difícil (pérdida de una estructura morfológica aparentemente adaptativa).

Para la identificación de las concepciones y patrones explicativos se aplicó una versión del método comparativo constante (GLASSER; STRAUSS, 1967), basado en la teoría fundamentada (grounded theory) (HERNÁNDEZ SAMPIERI; FERNÁNDEZ COLLADO; BAPTISTA LUCIO, 2010). Este método permite generar categorías teóricas a partir de los datos. En nuestro caso, se postula que estas categorías constituyen buenas caracterizaciones de las concepciones utilizadas por los estudiantes en sus explicaciones.

Cuadro 1. Consignas de los problemas utilizados para indagar las concepciones de los estudiantes. Abreviaturas: PP: problema de los piojos; PF: problema de los peces; PL: problema de los leones

PP: La siguiente noticia apareció en un diario: "El 15\% de los niños de las escuelas tuvieron piojos en el último año. La causa exacta de las recientes epidemias es desconocida, dado que las medidas de higiene se han incrementado, pero todos coinciden en que los insecticidas ya no tiene efecto sobre los piojos.” ¿Por qué crees que los insecticidas que se utilizaban con éxito anteriormente ya no tienen efecto?

PF: En un lago existe una población de peces que ha sido estudiada desde hace muchas décadas por un grupo de biólogos. Comparando las poblaciones más antiguas con las actuales los investigadores observaron un cambio en las aletas de los peces: la gran mayoría de los individuos de las poblaciones antiguas tiene aletas sin espinas, mientras que en las poblaciones actuales la mayoría de los individuos tiene aletas con espinas. Los investigadores observaron que las espinas eran una eficaz defensa contra sus depredadores. (a) ¿Cómo crees que explicaría un biólogo esta diferencia entre las poblaciones antiguas y las actuales? Hace unos años, debido a lluvias inusualmente fuertes, el nivel del lago subió, permitiendo que algunos peces ingresaran en un lago ubicado en un cueva a orillas del lago principal. Al bajar las aguas el lago de la cueva quedó aislado del lago principal. A la cueva no llega la luz solar, por lo que estos peces sobrevivieron en un ambiente totalmente oscuro. Cuando los biólogos estudiaron esta población de la cueva - años después- encontraron que estaba formada principalmente por individuos ciegos (tenían ojos muy pequeños y recubiertos con piel). (b) ¿Cómo podrías explicar, desde un punto de vista científico, por qué la población de las cuevas está formada principalmente por individuos ciegos?

PL: Una población de leones sufrió varias epidemias de moquillo (una enfermedad producida por un virus). En las primeras epidemias el porcentaje de leones infectados que murió fue muy alto, pero este porcentaje se redujo notablemente a través de las generaciones (a pesar de que el virus permaneció inalterado a través de los años) de modo que en las últimas epidemias sólo unos pocos leones murieron ¿Por qué crees que el virus ya no tiene el mismo efecto mortal en los leones? ¿Cómo podrías explicar este cambio?

Fuente: elaborado por los autores. 
Para la identificación de los obstáculos se tuvieron en cuenta, además de las concepciones y patrones explicativos identificados, los estudios en didáctica, historia y epistemología de la biología y - muy especialmente - investigaciones en psicología cognitiva. Estas fuentes nos permitieron proponer posibles obstáculos subyacentes a las concepciones. La identificación de obstáculos supone un ejercicio hermenéutico y está (comparativamente) más orientada por la teoría que la primera instancia de análisis. El carácter de obstáculo de los modos de razonamiento - cuya existencia propusimos - se evaluó de acuerdo con dos criterios: (1) los obstáculos son funcionales (sirven para dar cuenta de ciertos aspectos del mundo) y (2) nunca desaparecen. Así, si los modos de razonamiento identificados podían considerarse obstáculos se esperaría que, tras su aparente superación en la instancia post-instruccional, reaparecieran en dos circunstancias: (a) frente a problemas especialmente complejos en los que se dificulta la aplicación del modelo científico aprendido y (b) con el paso del tiempo. Con el propósito de evaluar la predicción (1) en la instancia inmediatamente post-instruccional los alumnos resolvieron un problema con un grado de dificultad medio [PF parte (a), equivalente al PP] y otro problema [PF parte (b)] de mayor complejidad. Con respeto a la segunda predicción los estudiantes resolvieron un problema (PL) de dificultad media un año después de la implementación de la UD.

\section{Resultados y discusión}

\section{Concepciones y patrones explicativos que los estudiantes utilizan para dar cuenta de la evolución adaptativa}

El análisis del amplio corpus empírico obtenido mediante la aplicación de los instrumentos mencionados nos permitió identificar numerosas concepciones que los estudiantes utilizan para dar cuenta de la evolución adaptativa en la instancia pre-instruccional. Como puede observarse en el Cuadro 2, algunas concepciones son más frecuentes que otras.

Ofrecemos a continuación un breve análisis de algunas de las concepciones identificadas. Privilegiamos aquellos casos cuyo análisis supone algún elemento novedoso en relación con estudios previos sobre el tema. La noción de "cambio individual adaptativo" (CIA) (Cuadro 2) aparece en un alto porcentaje de respuestas (se incluyeron en esta categoría, entre otras, las que apelan a la noción de "acostumbramiento"). Esta concepción, detectada en otras investigaciones (BARDAPURKAR, 2008; EVANS, 2010), es frecuentemente citada como un componente de las explicaciones "lamarckianas". Sin embargo, esta idea no resulta plausible para todos los estudiantes [Bardapurkar (2008) también reporta este hecho] y para otros es tan probable como otra noción alternativa que hemos denominado "crías adaptadas" (Cuadro 2). A partir de las entrevistas sugerimos que el hecho de que el CIA resulte plausible para un estudiante depende fuertemente del ejemplo analizado. 
Galli, L. G.; Meinardi, E.

Cuadro 2. Concepciones utilizadas por los estudiantes para explicar la evolución adaptativa

\begin{tabular}{|c|c|c|c|}
\hline Concepción & $\begin{array}{l}\text { Breve definición } \\
\text { de la concepción }\end{array}$ & $\begin{array}{l}\text { Ejemplo de respuesta en que } \\
\text { aparece la concepción }\end{array}$ & $\begin{array}{c}\text { Porcentaje } \\
\text { de respuestas } \\
\text { en las que } \\
\text { aparece }\end{array}$ \\
\hline $\begin{array}{l}\text { 1. Cambio } \\
\text { individual } \\
\text { adaptativo }\end{array}$ & $\begin{array}{l}\text { Los individuos sufren } \\
\text { transformaciones } \\
\text { adaptativas al enfrentar un } \\
\text { problema ambiental. }\end{array}$ & $\begin{array}{l}\text { Los insecticidas que se utilizaban } \\
\text { antes ya no tienen efecto sobre } \\
\text { los piojos porque los bichos } \\
\text { son resistentes ya que se } \\
\text { 'acostumbran' a estos tipos de } \\
\text { 'medicamentos' }[\ldots]\end{array}$ & 83,3 \\
\hline 2. Crías adaptadas & $\begin{array}{l}\text { Los individuos no } \\
\text { sufren transformaciones } \\
\text { adaptativas pero producen } \\
\text { crías que nacen con } \\
\text { los rasgos adaptativos } \\
\text { necesarios para sobrevivir. }\end{array}$ & $\begin{array}{l}\text { Como que a medida que se van } \\
\text { procreando nacen con defensas } \\
\text { para resistir. }\end{array}$ & 52,1 \\
\hline $\begin{array}{l}\text { 3. Negación } \\
\text { variacional }\end{array}$ & $\begin{array}{l}\text { Se considera poco probable } \\
\text { que nazca un individuo con } \\
\text { un rasgo que no resulta } \\
\text { necesario para sobrevivir. }\end{array}$ & $\begin{array}{l}\text { E- Si no se usara el insecticida } \\
\text { ¿Tu crees que igual podría nacer } \\
\text { una cría resistente al insecticida? } \\
\text { A- No, porque nuca se le aplicó. }\end{array}$ & 52,1 \\
\hline $\begin{array}{l}\text { 4. Finalismo } \\
\text { natural }\end{array}$ & $\begin{array}{l}\text { Los procesos biológicos } \\
\text { están orientados a ciertas } \\
\text { metas de un modo } \\
\text { "natural", esto es, no } \\
\text { obedecen ni a la intención } \\
\text { de los organismos ni a la de } \\
\text { alguna entidad sobrenatural. }\end{array}$ & $\begin{array}{l}\text { E- }[\ldots] \text { ¿Cómo crees tu que los } \\
\text { piojos logran sufrir justamente } \\
\text { esos cambios que necesitan para } \\
\text { sobrevivir? ¿Crees que ellos } \\
\text { pueden se algún modo dirigir } \\
\text { esos cambios que sufren? A- } \\
\text { No, son naturales, es natural del } \\
\text { organismo. }\end{array}$ & 48 \\
\hline $\begin{array}{l}\text { 5. Finalismo } \\
\text { implícito }\end{array}$ & $\begin{array}{l}\text { Aunque no se explicita, se } \\
\text { asume que el cambio está } \\
\text { orientado a alcanzar una } \\
\text { meta predeterminada. }\end{array}$ & $\begin{array}{l}\text { Las condiciones del entorno } \\
\text { cambian constantemente, por lo } \\
\text { tanto también los mecanismos } \\
\text { que antes tenía el ser vivo para } \\
\text { mantenerse dentro del mismo } \\
{[\ldots]}\end{array}$ & 35,4 \\
\hline $\begin{array}{l}\text { 6. Herencia de } \\
\text { los caracteres } \\
\text { adquiridos }\end{array}$ & $\begin{array}{l}\text { Aquellas transformaciones } \\
\text { adaptativas sufridas por los } \\
\text { individuos son heredadas } \\
\text { por sus descendientes. }\end{array}$ & $\begin{array}{l}{[\ldots] \text { si se hizo resistente, el hijo }} \\
\text { supongo que va a nacer resistente } \\
\text { ya }[\ldots]\end{array}$ & 54,1 \\
\hline
\end{tabular}

continua 
Cuadro 2. continuação

\begin{tabular}{|c|c|c|c|}
\hline Concepción & $\begin{array}{l}\text { Breve definición } \\
\text { de la concepción }\end{array}$ & $\begin{array}{l}\text { Ejemplo de respuesta en que } \\
\text { aparece la concepción }\end{array}$ & $\begin{array}{c}\text { Porcentaje } \\
\text { de respuestas } \\
\text { en las que } \\
\text { aparece }\end{array}$ \\
\hline $\begin{array}{l}\text { 7. Variabilidad } \\
\text { previa }\end{array}$ & $\begin{array}{l}\text { Existen diferencias } \\
\text { interindividuales anteriores } \\
\text { a la existencia de la presión } \\
\text { de selección. }\end{array}$ & $\begin{array}{l}\text { Sí, pero que igual hay diferencias } \\
\text { entre los piojos, como que no } \\
\text { son todos iguales y unos capaz } \\
\text { tienen más resistencia que otros y } \\
\text { por eso pudieron sobrevivir, pero } \\
\text { no que nacieron directamente } \\
\text { siendo inmunes. }\end{array}$ & 52,1 \\
\hline $\begin{array}{l}\text { 8. Relación } \\
\text { variante - fitness }\end{array}$ & $\begin{array}{l}\text { Existe una relación } \\
\text { entre las variantes y las } \\
\text { probabilidades de sobrevivir } \\
\text { y reproducirse. }\end{array}$ & $\begin{array}{l}{[\ldots] \text { algún piojo tipo } \mathrm{X} \text { es inmune }} \\
\text { por alguna causa biológica a este } \\
\text { insecticida. Todos los demás } \\
\text { murieron menos los piojos X } \\
{[\ldots]}\end{array}$ & 43,7 \\
\hline $\begin{array}{l}\text { 9. Cambio } \\
\text { poblacional }\end{array}$ & $\begin{array}{l}\text { Las proporciones de las } \\
\text { variantes en la población } \\
\text { cambian como consecuencia } \\
\text { de las diferencias en las } \\
\text { probabilidades de sobrevivir } \\
\text { y reproducirse asociadas a } \\
\text { cada variante. }\end{array}$ & $\begin{array}{l}{[\ldots] \text { a ciertos piojos el insecticida }} \\
\text { no los afecta estos sobreviven } \\
\text { y los demás mueren, los que } \\
\text { sobreviven se reproducen y son } \\
\text { cada vez más, así con el tiempo el } \\
\text { insecticida se vuelve inservible. }\end{array}$ & 12,5 \\
\hline $\begin{array}{l}\text { 10. Analogía } \\
\text { adaptación } \\
\text { evolutiva - } \\
\text { adaptación } \\
\text { fisiológica }\end{array}$ & $\begin{array}{l}\text { La evolución adaptativa (ej.: } \\
\text { evolución de la resistencia } \\
\text { a pesticidas) es como la } \\
\text { adaptación fisiológica de los } \\
\text { individuos (ej.: la inmunidad } \\
\text { adquirida mediante la } \\
\text { vacunación). }\end{array}$ & $\begin{array}{l}\text { Creo que debería evolucionar } \\
\text { todo el tiempo el insecticida } \\
\text { porque siempre deja de funcionar } \\
\text { en algún momento. Es como } \\
\text { cuando uno abusa de un remedio, } \\
\text { cada vez siente menos }[\ldots]\end{array}$ & 33,3 \\
\hline $\begin{array}{l}\text { 11. Extinción por } \\
\text { cambio brusco }\end{array}$ & $\begin{array}{l}\text { La causa de la extinción } \\
\text { de un linaje es siempre un } \\
\text { cambio ambiental muy } \\
\text { brusco. }\end{array}$ & $\begin{array}{l}\text { E- La hipótesis más aceptada } \\
\text { es que lo que pasó es que cayó } \\
\text { un meteorito en la Tierra que } \\
\text { produjo una serie de cambios } \\
\text { climáticos importantes... A- Sí, } \\
\text { puede ser, claro, pero es como } \\
\text { que tiene que haber así, un hecho } \\
\text { re, que cambie todo... }\end{array}$ & 23 \\
\hline
\end{tabular}

continua 
Cuadro 2. continuação

\begin{tabular}{|c|c|c|c|}
\hline Concepción & $\begin{array}{l}\text { Breve definición } \\
\text { de la concepción }\end{array}$ & $\begin{array}{l}\text { Ejemplo de respuesta en que } \\
\text { aparece la concepción }\end{array}$ & $\begin{array}{l}\text { Porcentaje } \\
\text { de respuestas } \\
\text { en las que } \\
\text { aparece }\end{array}$ \\
\hline $\begin{array}{l}\text { 12. Extinción } \\
\text { antropogénica }\end{array}$ & $\begin{array}{l}\text { La causa de la extinción de } \\
\text { un linaje es la intervención } \\
\text { humana en el ambiente. }\end{array}$ & $\begin{array}{l}\text { E- Tu habrás oído decir que } \\
\text { muchas especies de seres vivos } \\
\text { se han extinguido. A- Y... pero } \\
\text { porque la mano del hombre } \\
\text { estuvo presente ahí, de manera } \\
\text { muy drástica }[. . .]\end{array}$ & 21 \\
\hline $\begin{array}{l}\text { 13. Variación } \\
\text { azarosa }\end{array}$ & $\begin{array}{l}\text { Las variantes surgen de un } \\
\text { modo independiente de su } \\
\text { valor adaptativo. }\end{array}$ & $\begin{array}{l}{[\ldots] \text { Para mí es azaroso, o sea, }} \\
\text { no viene de la elección de uno } \\
\text { mismo si no si está capacitado o } \\
\text { no }[\ldots]\end{array}$ & 14,6 \\
\hline $\begin{array}{l}\text { 14. Finalismo } \\
\text { explícito }\end{array}$ & $\begin{array}{l}\text { Se explicita que los cambios } \\
\text { están orientados a alcanzar } \\
\text { una meta predeterminada. }\end{array}$ & $\begin{array}{l}\text { Creo que el principal objetivo } \\
\text { de un ser vivo es, justamente, } \\
\text { sobrevivir. }\end{array}$ & 14,6 \\
\hline $\begin{array}{l}\text { 15. Inducción } \\
\text { ambiental }\end{array}$ & $\begin{array}{l}\text { El entorno induce } \\
\text { directamente el cambio } \\
\text { adaptativo de los individuos. }\end{array}$ & $\begin{array}{l}\text { Sí, pero porque lo tiene en su } \\
\text { cuerpo, su cuerpo reacciona así, } \\
\text { no su cabeza que él piensa ‘¿Qué } \\
\text { puedo hacer para zafar de este } \\
\text { producto?' ¿Me entiendes? Tipo } \\
\text { una reacción que tiene él es que } \\
\text { tiene el insecticida ya impregnado } \\
\text { y cuando le tirás más no le hace } \\
\text { nada porque ya lo tiene, no es } \\
\text { que lo tiene que pensar eso ¿Me } \\
\text { entiendes?”. }\end{array}$ & 8,33 \\
\hline
\end{tabular}

continua

Otro aspecto destacable es la alta frecuencia con que aparece la noción de "variabilidad previa" (Cuadro 2). Esta es una componente fundamental de las explicaciones darwinianas y fue encontrada en más del cincuenta por ciento de las respuestas. Sin embargo, solo un doce por ciento de las respuestas es de tipo "seleccional" (cercana al MESN). Esto sugiere que un problema importante para que los estudiantes construyan el MESN podría ser que no reconocen la pertinencia de la noción de variabilidad para explicar el cambio adaptativo, a diferencia de lo que se sugiere en otras investigaciones respecto de que carecen de dicha noción. Esta interpretación coincide con lo señalado por Greene (1990). Cabe mencionar que en la instancia escrita muy pocos estudiantes (un 16\%) recurrieron espontáneamente a esta noción y que el porcentaje fue mayor $(50 \%)$ cuando durante la entrevista se les preguntó explícitamente por 
Cuadro 2. continuação

\begin{tabular}{|c|c|c|c|}
\hline Concepción & $\begin{array}{l}\text { Breve definición } \\
\text { de la concepción }\end{array}$ & $\begin{array}{l}\text { Ejemplo de respuesta en que } \\
\text { aparece la concepción }\end{array}$ & $\begin{array}{c}\text { Porcentaje } \\
\text { de respuestas } \\
\text { en las que } \\
\text { aparece }\end{array}$ \\
\hline 16. Compensación & $\begin{array}{l}\text { La pérdida evolutiva de } \\
\text { un rasgo se explica por la } \\
\text { adquisición simultánea de } \\
\text { otro rasgo de mayor valor } \\
\text { adaptativo }\end{array}$ & $\begin{array}{l}\text { Azarosamente algunos peces } \\
\text { nacieron con los ojos un poquito } \\
\text { más pequeños y recubiertos con } \\
\text { una leve capa de piel, que aún les } \\
\text { dejaba ver y con un olfato apenas } \\
\text { más agudo, estos tenían más } \\
\text { chances de sobrevivir ya que con } \\
\text { el olfato encontraban el alimento. }\end{array}$ & 36 \\
\hline $\begin{array}{l}\text { 17. Mutación } \\
\text { dirigida }\end{array}$ & $\begin{array}{l}\text { La probabilidad de } \\
\text { ocurrencia de una mutación } \\
\text { está positivamente } \\
\text { correlacionada con su valor } \\
\text { adaptativo }\end{array}$ & $\begin{array}{l}\text { Es más probable que esta } \\
\text { mutación ocurra en el caso de } \\
\text { Daniela ya que, con la presencia } \\
\text { de un antibiótico contra ellas, } \\
\text { las bacterias sensibles pueden } \\
\text { muy difícilmente desarrollar } \\
\text { inmunidad y así pasar de } \\
\text { generación en generación. Sin la } \\
\text { presencia del antibiótico no hay } \\
\text { razón por la cual inmunizarse, } \\
\text { entonces no muta. }\end{array}$ & 50 \\
\hline
\end{tabular}

Abreviaturas: E: entrevistador; A: alumno/a.

Fuente: elaborado por los autores.

la existencia de variabilidad. Este hecho es coherente con la hipótesis de que la existencia de variabilidad es concebida (o fácilmente concebible) por muchos estudiantes que, sin embargo, no recurren espontáneamente a ella por no considerarla relevante para explicar el problema planteado. Este análisis sugiere la necesidad de revisar la conclusión según la cual un problema fundamental para la enseñanza del MESN es que los estudiantes carecen de la noción de variabilidad (ANDERSSON; WALLIN, 2006; PRINOU; HALKIA; SKORDOULIS 2008).

La noción de "herencia de los caracteres adquiridos" (HCA) (Cuadro 2) aparece en poco más que el cincuenta por ciento de las respuestas. Esta idea es también frecuentemente mencionada en la literatura didáctica como parte de las concepciones "lamarckianas" JIMÉNEZ ALEIXANDRE, 1991). Al igual que lo señalado para el CIA, también en este caso consideramos probable que el ejemplo analizado influya significativamente en las respuestas de los estudiantes ya que durante las entrevistas muchos mostraron cierta desconfianza de esta noción.

Otra característica de las concepciones de los estudiantes frecuentemente citada es su carácter "vitalista" o "intencional", asociado a una perspectiva antropomórfica (SETTLAGE, 1994; SINATRA; BREM; EVANS, 2008). En nuestro trabajo hemos hallado que, por el con- 
trario, muchos estudiantes tienen una visión teleológica de la evolución sin connotaciones antropomórficas, intencionales o vitalistas evidentes: denominamos a esta idea "finalismo natural" (Cuadro 2). A partir de este hallazgo sugerimos la necesidad de reconsiderar la problemática relación entre teleología, intencionalidad, vitalismo, animismo y antropomorfismo. Así, casi un cincuenta por ciento de los estudiantes consideró que los cambios biológicos se orientan a la adaptación en virtud de una tendencia "natural" derivada de la organización de los seres vivos y no debido a una entidad intencional.

La concepción que hemos denominado "compensación” (Cuadro 2) también merece algún análisis ya que aunque nuestros datos muestran su frecuente aparición (un 36\% en la instancia post-instruccional) prácticamente no se menciona en la literatura en didáctica 5 . El hecho de que muchos estudiantes asuman que la pérdida de un rasgo supone necesariamente la adquisición de otro "mejor" nos permite hacer algunas inferencias sobre el modo en que conciben la evolución. En primer lugar, cabe suponer que para los estudiantes la pérdida de un rasgo es siempre algo negativo. Saben, por otro lado, que la evolución por selección natural supone que el rasgo seleccionado debe implicar una ventaja. Por eso, para explicar la pérdida de una estructura mediante el MESN deben suponer que la misma implica el origen de otro rasgo ventajoso. Por otro lado, este razonamiento evidencia que los estudiantes no disponen del concepto -central- de "compromiso" (trade off), según el cual todos los rasgos, incluso aquellos selectivamente ventajosos, implican costos. Comprender el MESN supone entender que aquellas variantes de un rasgo que son seleccionadas lo son en virtud de que los beneficios que suponen en relación con el fitness superan los costos. Tener presente este rasgo del modelo y el mencionado sesgo de los razonamientos de los estudiantes ayuda a diseñar actividades que propicien la discusión explícita de la noción de "compromiso". Cabe señalar que la explicación de la pérdida de la visión constituye un caso de difícil explicación aún para los biólogos (ESPINASA; ESPINASA, 2008) y también para el profesorado de biología, como se detectó en un trabajo anterior (MEINARDI; ADÚRIZ-BRAVO, 2002).

$\mathrm{El}$ análisis de las respuestas nos permitió identificar dos grandes patrones explicativos que denominamos "finalista" y "seleccional". Metodológicamente, los patrones explicativos se definieron en función de las concepciones puestas en juego en las explicaciones ${ }^{6}$. El patrón "finalista" fue el más frecuente $(87,5 \%$ de las respuestas) en el cual distinguimos dos variantes. La primera ("adaptación directa por cambio individual") supone que frente a un problema ambiental los individuos se transforman en sentido adaptativo y que dicha transformación es heredada por la descendencia mientras que, según la otra variante ("adaptación indirecta por crías adaptadas"), los individuos no se transforman en sentido adaptativo sino que producen crías adaptadas. Un ejemplo del primer caso es el siguiente: "Los insecticidas que se utilizaban antes ya no tienen efecto sobre los piojos porque los bichos son resistentes ya que se 'acostumbran' a estos tipos de

\footnotetext{
${ }^{5}$ La única referencia a una concepción semejante que hemos encontrado en la bibliografía es Nehm y Reilly (2007).

${ }^{6}$ Se consideraron "finalistas" aquellas respuestas que incluían al menos un incidente correspondiente a las concepciones 1, 2, 3, 5, 14 o 15 (cuadro 2). Las respuestas que incluían incidentes de las concepciones 7 y 8 (Cuadro 2), en ausencia de las categorías indicadoras de finalismo, se consideraron "seleccionales".
} 
'medicamentos' [...]". El segundo caso es ilustrado por la siguiente respuesta: "A medida que van procreando nacen con defensas para resistir".

El patrón "seleccional" supone que frente a un problema ambiental sobreviven y se reproducen con más probabilidad aquellos individuos que poseen alguna característica que les confiere alguna ventaja frente al problema ambiental. Este patrón fue menos frecuente $(12,5 \%$ de las respuestas) y puede ilustrarse con la siguiente respuesta:

Tal vez el insecticida mataba ocho de cada diezpiojos por características genéticas
de estos. Entonces morirían los piojos vulnerables al producto y sobrevivirían los
demás. Los sobrevivientes se reproducian y transmitían su inmunidad al insecticida
a sus descendientes mediante sus genes. De esta forma el producto se volvería cada
vez. menos efectivo.

Consideramos que este patrón explicativo es cercano al MESN.

Algunas respuestas pueden interpretarse como la expresión de modelos sintéticos (VOSNIADOU, 2006, 2012) o de distintos modelos teóricos coexistentes (POZO; GÓMEZ CRESPO, 2004). Por ejemplo, según algunos estudiantes al aplicarse un insecticida a una población de piojos solo sobreviven aquellos que "fueron capaces de adaptarse". Esta respuesta combina un elemento de las explicaciones finalistas (los individuos se adaptan) con un elemento de las explicaciones seleccionales (solo algunos logran dicha adaptación).

\section{Obstáculos subyacentes}

A partir de nuestro análisis proponemos la existencia de, al menos, tres obstáculos subyacentes que hemos denominado "teleología de sentido común" (TSC), "razonamiento centrado en el individuo" (RCI) y "razonamiento causal lineal” (RCL). Definimos la TSC como un modo de pensar según el cual todas las estructuras y procesos biológicos están orientados a la consecución de un fin. El RCI designa el razonamiento según el cual los procesos biológicos (incluida la evolución) tienen lugar a nivel individual y, finalmente, denominamos RCL al razonamiento según el cual todo fenómeno tiene una causa única y directa que lo precede temporalmente.

Estos modos de razonar se consideraron obstáculos de acuerdo con los criterios definidos en la introducción (transversalidad, funcionalidad y competencia). En el análisis se tuvieron en cuenta evidencias provenientes de la investigación en didáctica, epistemología e historia de la biología y de la psicología cognitiva. Solo ofrecemos aquí dos breves comentarios al respecto.

En particular, son relevantes las evidencias provenientes de la psicología cognitiva en relación con la TSC y con el RCL. Numerosos investigadores sostienen que la visión teleológica del mundo biológico es parte constitutiva de la cognición normal (EVANS, 2010; INAGAKI; HATANO, 2010; KEIL, 2002; KELEMEN, 1999). En relación con el dominio biológico, este sesgo cognitivo estaría presente en todas las edades, al menos desde los dos años de vida (CASLER; KELEMEN, 2007) y hasta la adultez (KELEMEN; ROSSET, 2009). Estas investigaciones destacan la funcionalidad cognitiva del razonamiento teleológico (su poder heurístico y predictivo) y muchos autores sugieren incluso que este sesgo cognitivo posee un fundamento biológico. Por otro lado, se trata de un modo de pensar que las personas aplican a los más di- 
versos ámbitos de contenido (transversalidad), aunque el dominio biológico constituye en los adultos un área privilegiada de aplicación. Esta perspectiva intuitiva permite a las personas dar sentido a muchos fenómenos, incluida la adaptación evolutiva que esperamos los estudiantes expliquen apelando al MESN (competencia).

Del mismo modo, el RCL es citado como un sesgo cognitivo universal y transversal ya que aparece en razonamientos ligados no solo al ámbito de la biología sino a prácticamente todas las áreas de contenido (DRIVER; GUESNE; TIBERGHIEN, 1992; POZO, 1999; POZO; GÓMEZ CRESPO, 2004; TALANQUER, 2006). También se trata de un razonamiento funcional ya que permite identificar algunas de las causas de los fenómenos, con el poder predictivo que esto supone. Muchos modelos científicos (entre ellos el MESN) suponen considerar la acción simultánea de muchos factores causales (POZO; GÓMEZ CRESPO, 2004). En el caso de la evolución adaptativa, el RCL sugiere que el cambio es una consecuencia directa de algún factor ambiental (ver categoría 15 en el Cuadro 2). Así, el RCL compite con el MESN, ofreciendo al estudiante una explicación más sencilla y próxima a la intuición.

El tercer obstáculo identificado, el razonamiento centrado en el individuo (RCI) sería el menos transversal. En general, se señala que los estudiantes carecen de la perspectiva poblacional requerida para la comprensión del MESN. Esta perspectiva resalta lo que el estudiante no tiene. Proponemos que, sin negar esta conclusión, puede ser útil considerar que el pensamiento en términos individuales es una estrategia cognitiva positiva ( $\mathrm{y}$ no meramente la evidencia de una falta) basada en la analogía entre la adaptación fisiológica y la evolutiva. Suponer que el cambio evolutivo adaptativo se reduce al cambio fisiológico de los individuos permite dar cuenta del primer fenómeno (funcionalidad) sin recurrir al MESN (competencia). Es reconocido el hecho de que la analogía constituye un mecanismo cognitivo de gran importancia (LEHRER; SCHAUBLE, 2006; NERSESSIAN, 1992). En el caso que nos ocupa sugerimos que para dar cuenta de la adaptación evolutiva (poblacional) muchos estudiantes establecen una analogía con la adaptación fisiológica (individual) (ver la categoría 10 en el Cuadro 2). Eso podría explicar por qué ciertas concepciones resultan plausibles en algunos casos y no en otros. Por ejemplo, para muchos estudiantes el cambio individual adaptativo es concebible en el caso de la resistencia a un patógeno pero no en el caso de una transformación morfológica como podría ser la aparición de plumas. Esto podría explicarse si se supone que estos estudiantes establecieron una analogía entre la evolución de la resistencia a un patógeno y la inmunización de los individuos mediante la vacunación. Desde esta perspectiva, la experiencia cotidiana sugiere que la fisiología puede cambiar adaptativamente pero no así la morfología.

Postulamos que estos tres obstáculos dan cuenta, al menos parcialmente, de las concepciones y patrones explicativos identificados. Tal como señalamos, a partir de esta hipótesis predecimos que, dada la persistencia de los obstáculos, las mismas concepciones y explicaciones reaparecerían frente a problemas de alta complejidad y con el paso del tiempo. Consideramos que el patrón explicativo finalista es la expresión de estos tres obstáculos, por lo que esperamos que las explicaciones finalistas incrementaran su frecuencia en las instancia post-instruccionales en dos circunstancias: (1) al enfrentar problemas más complejos en la instancia inmediatamente posterior a la aplicación de la UD; y (2) al enfrentar problemas de complejidad media un año después de la implementación de la UD. A continuación presentamos los datos que apoyan estas predicciones. 
Cabe hacer un comentario adicional sobre la relación entre la TSC (GONZÁLEZ GALLI; MEINARDI, 2006, 2008, 2011) y el MESN ya que mientras en el caso de los otros dos obstáculos identificados (RCL y RCI) es claro que son incompatibles con el MESN, ciertos análisis epistemológicos sugieren que esto no es evidente en el caso de la TSC. En los análisis didácticos suele afirmarse que la teleología es un rasgo de las concepciones de los estudiantes al tiempo que se asume que este modo de pensar no tiene ningún rol legítimo en la biología "erudita". Sin embargo, no solo puede argumentarse que los razonamientos teleológicos cumplen un rol heurístico en la biología académica sino que varios autores (AYALA, 1999; CAPONI, 2003; DENNETT, 1991; LENNOX, 1993; RUSE, 2000, entre otros) sostienen que las explicaciones basadas en el MESN son en sí mismas teleológicas. Aunque esta afirmación es muy discutida, creemos necesario reconocer, al menos, el carácter problemático del estatus de la teleología en la biología. Cabe destacar que el eventual reconocimiento del carácter teleológico del MESN no implica afirmar que los razonamientos de los estudiantes son iguales a aquellos basados en dicho modelo científico. De hecho, las diferencias son fundamentales: mientras que el "nexo teleológico" en las explicaciones darwinianas residiría en la relación entre el aumento de frecuencia de la variante seleccionada y la "presión de selección" (CAPONI, 2003) en el caso de las explicaciones de los estudiantes este nexo reside en la relación entre dicha presión y el origen de las variantes en la población. A pesar de esta diferencia fundamental, en ambos casos se estaría apelando a un razonamiento teleológico. Ese análisis sugiere la necesidad de analizar críticamente la tendencia a censurar toda forma de análisis teleológico en las clases de biología (ZOHAR; GINOSSAR, 1998). Nos alejamos así de los supuestos según los cuales la biología no presenta ninguna forma de teleología, postura desde la que se suele inferir que las concepciones teleológicas de los estudiantes se deben (y pueden) eliminar.

\section{Análisis comparativo pre / post-instruccional}

En la Tabla 1 puede observase que la frecuencia de las explicaciones seleccionales se incrementó notablemente tras la implementación de la UD. Comparando los valores de las filas segunda y tercera se evidencia que frente a un problema de mayor dificultad (PF, punto b) tiene lugar cierta "reversión” al pensamiento finalista. Este resultado sugiere que los obstáculos persisten después de la instrucción y se vuelven a "activar" cuando los nuevos marcos teóricos aprendidos (el MESN) resultan de difícil aplicación. Esto es lo que cabría esperar si las TSC, el RCL y el RCI funcionaran como un obstáculos ya que, en rigor, estos nunca desaparecen (ASTOLFI, 1994). Por otro lado, el relativamente bajo porcentaje de respuestas finalistas aún en el punto (b) del problema de los peces (PF) evidencia la potencia de la UD centrada en la superación de la TSC.

Los resultados obtenidos para el problema de los leones (PL) (Tabla 1), un año después de la implementación de la UD, también abonan la hipótesis de que la TSC, el RCL y el RCI funcionan como obstáculos en sentido estricto. En efecto, en esta instancia un mayor porcentaje de estudiantes ha recurrido a explicaciones finalistas en comparación con la instancia inmediatamente posterior a la implementación de la UD (PF, punto a). Esto refleja cierta inestabilidad temporal en el modelo aprendido y la persistencia y disponibilidad de los obstáculos identificados. Esta leve reducción de las respuestas "seleccionales" después de un año se da 
junto con un incremento de respuestas "finalistas" y no de otro tipo ("otras" o "no responde"), hecho que interpretamos como una evidencia de que los obstáculos persisten y conservan su funcionalidad en la estructura cognitiva de los estudiantes.

Tabla 1. Frecuencia de los tipos de explicaciones en las instancias pre y post-instruccional

\begin{tabular}{lccc}
\hline \multirow{2}{*}{ Instancia de indagación } & \multicolumn{3}{c}{ Porcentaje de respuestas } \\
\cline { 2 - 4 } & Finalista & Seleccional & Otros \\
\hline Pre-instruccional (PP + entrevista) & 87,5 & 12,5 & 0 \\
Post-instruccional & & & \\
a corto plazo (PF, punto a) & 10 & 88 & 2 \\
a corto plazo (PF, punto b) & 32 & 68 & 0 \\
a largo plazo (PL, punto a) & 24 & 76 & 0 \\
\hline
\end{tabular}

Fuente: elaborado por los autores.

\section{Implicancias didácticas y conclusiones}

El enfoque cualitativo de nuestra investigación nos permitió caracterizar en detalle el modo en que los estudiantes de dos cursos de escuela secundaria explican la evolución adaptativa. Se identificaron numerosas concepciones y los principales patrones explicativos. A partir de estos análisis postulamos la existencia de tres obstáculos subyacentes a las concepciones detectadas. Hemos denominado a estos obstáculos "teleología de sentido común", "razonamiento centrado en el individuo" y "razonamiento causal lineal".

Las evidencias provenientes de nuestra investigación y el marco teórico adoptado (obstáculos) nos llevan a sugerir que no es posible (ni deseable) la eliminación y reemplazo de los obstáculos tal como se sostiene desde diversas posturas relacionadas con el "cambio conceptual" (DUIT, 2006; SCOTT; ASOKO; LEACH, 2007). Así, sugerimos que el principal objetivo didáctico en relación con los obstáculos consiste en el desarrollo de una "vigilancia" de tipo metacognitivo. Este resultado es convergente con numerosas investigaciones que tienden a señalar que el desarrollo de las destrezas metacognitivas constituye una condición indispensable para el aprendizaje de los modelos científicos (THOMAS, 2012; VEENMAN, 2012; ZOHAR; DORI, 2012). Esta necesidad del trabajo metacognitivo sería especialmente relevante en relación con los razonamientos teleológicos debido al problemático estatus del mismo en la propia biología. Esto es, si se reconoce que la teleología cumple un rol legítimo en la propia biología no se puede pretender su eliminación durante el aprendizaje escolar. En cambio, el objetivo sería que los estudiantes pudieran identificar este tipo de razonamiento y tomar conciencia de su potencial heurístico y explicativo así como de sus limitaciones, evaluación esta que debería ser fuertemente dependiente del contexto. Así, nuestras conclusiones plantean algunas diferencias en comparación con otras investigaciones en relación con la naturaleza y funcionamiento de ciertos modos de razonamiento (obstáculos) que subyacen a muchas concepciones particulares y también en relación con la naturaleza del modelo a enseñar. 
En síntesis, sugerimos que el trabajo orientado a la toma de conciencia por parte de los estudiantes de la existencia y funcionamiento de unos pocos modos de razonar muy generales - los obstáculos - podría ser un potente marco general para el diseño de estrategias didácticas que faciliten un progreso en la comprensión de los principios del modelo de evolución por selección natural.

\section{Referencias}

ADÚRIZ-BRAVO, A. A semantic view of scientific models for science education. Science \& Education, Dordrecht, v. 22, n. 7, p. 1593-1611, 2012.

AFFANATO, F. E. A survey of biology teachers' opinions about the teachings of evolutionary theory and/or the creation model in the United States in public and private schools. Dissertation (PhD) - University of Iowa, Iowa City, 1986.

ALTERS, B. J.; NELSON, C. E. Perspective: teaching evolution in higher education.

Evolution: international journal of organic evolution, Oxford, v. 56, n. 10, p. 1891-1901, 2002.

ANDERSSON, B.; WALLIN, A. On developing content-oriented theories taking biological evolution as an example. International Journal of Science Education, Abingdon, v. 28, n. 6, p. 673-695, 2006.

ASTOLFI, J. Conceptos clave en la didáctica de las disciplinas. Sevilla: Díada, 2001. . El trabajo didáctico de los obstáculos, en el corazón de los aprendizajes científicos. Enseñanza de las Ciencias, Barcelona, v. 12, n. 2, p. 206-216, 1994.

AYALA, F. Adaptation and novelty: teleological explanations in evolutionary biology. History and Philosophy of the Life Sciences, Heidelberg, v. 21, n. 1, p. 3-33, 1999.

BANET, E.; AYUSO, G. E. Teaching of biological inheritance and evolution of living beings in secondary school. International Journal of Science Education, Abingdon, v. 25, n. 3, p. 373-407, 2003.

BARDAPURKAR, A. Do students see the "selection" in organic evolution?: a critical review of the causal structure of student explanations. Evolution: education \& outreach, New York, v. 1, n. 3, p. 299-305, 2008.

BERKMAN, M. B.; PACHECO, J. S.; LUTZER, E. Evolution and creationism in America's classrooms: A national portrait. PLoS Biology, Cambridge, v. 6, n. 5, p. 920-924, 2008.

BISHOP, B. A.; ANDERSON, C. W. Student conceptions of natural selection and its role in evolution. Journal of Research in Science Teaching, Hoboken, v. 27, n. 5, p. 415-427, 1990. 
Galli, L. G.; Meinardi, E.

BRUMBI, M. N. Misconceptions about the concept of natural selection by medical biology students. Science Education, Hoboken, v. 68, n. 4, p. 493-503, 1984.

CAMILLONI, A. (Comp.). Los obstáculos epistemológicos en la enseñanza. Barcelona: Gedisa, 2001.

CAPONI, G. Darwin: entre Paley y Demócrito. História, Ciências, Saúde Manguinhos, Rio de Janeiro, v. 10, n. 3, p. 993-1023, 2003.

CASLER, K.; KELEMEN, D. Reasoning about artifacts at 24 months: the developing teleofunctional stance. Cognition, Amsterdam, v. 103, n. 1, p. 120-130, 2007.

DAGHER, Z. R.; BOUJAOUDE, S. Scientific views and religious beliefs of college students: the case of biological evolution. Journal of Research in Science Teaching, Hoboken, v. 34, n. 5, p. 429-445, 1997.

DEMASTES, S. S.; SETTLAGE JR., J.; GOOD, R. Students' conceptions of natural selection and its role in evolution: cases of replication and comparison. Journal of

Research in Science Teaching, Hoboken, v. 32, n. 5, p. 535-550, 1995.

DENNET'T, D. La actitud intencional. Barcelona: Gedisa, 1991.

DRIVER, R.; GUESNE, E.; TIBERGHIEN, A. Ideas científicas en la infancia y la adolescencia. Madrid: Morata, 1992.

DUIT, R. Enfoques del cambio conceptual en la enseñanza de las ciencias. In: SCHNOTZ, W.; VOSNIADOU, S.; CARRETERO, M. (Comp.). Cambio conceptual y educación. Buenos Aires: Aique, 2006. p. 219-249.

ESPINASA, M.; ESPINASA, L. Losing sight of regresive evolution. Evolution: education \& outreach, New York, v. 1, n. 4, p. 509-516, 2008.

EVANS, M. Conceptual change and evolutionary biology: a developmental analysis. In: VOSNIADOU, S. (Ed.). International handbook of research in conceptual change. New York: Routledge, 2010. p. 263-294.

FOLGUERA, G.; GONZÁLEZ GALLI, L. La extensión de la síntesis evolutiva y los alcances sobre la enseñanza de la teoría de la evolución. Bio-grafía: escritos sobre la Biología y su enseñanza, Bogotá, v. 5, n. 9, p. 4-18, 2012.

FUTUYMA, D. Evolution. 2. ed. Sunderland: Sinauer, 2009.

GLASER, B.; STRAUSS, A. The discovery of grounded theory: strategies for qualitative research. New York: Aldine Publishing, 1967.

GOETZ, J.; LECOMPTE, M. Etnografía y diseño cualitativo en investigación educativa. Madrid: Morata, 1988.

GONZÁLEZ GALLI, L.; MEINARDI, E. ¿Está en crisis el darwinismo?: los nuevos modelos de la biología evolutiva y sus implicaciones didácticas. Didáctica de las Ciencias Experimentales y Sociales, Valencia, n. 27, p. 219-234, 2013. 
Obstáculos para el aprendizaje del modelo ...

. Ni darwinianos ni lamarckianos: finalistas. In: JORNADAS NACIONALES, 8; CONGRESO INTERNACIONAL DE ENSEÑANZA DE LA BIOLOGÍA, 3., 2008, Mar del Plata. Memorias... Buenos Aires: ADBIA, 2008.

. Obstacles in the learning of natural selection. In: CONFERENCE OF EUROPEAN RESEARCHERS IN DIDACTICS OF BIOLOGY, 6., 2006, London. Proceedings... London: University of London, 2006.

. Revisión del concepto de obstáculo a partir de la investigación sobre la enseñanza y el aprendizaje del modelo de evolución por selección natural. In: JORNADAS NACIONALES, 9; IV CONGRESO INTERNACIONAL DE ENSEÑANZA DE LA BIOLOGÍA, 4., 2010, San Miguel de Tucumán. Memorias... Buenos Aires: ADBIA, 2010.

The role of teleological thinking in learning the darwinian model of evolution.

Evolution: education \& outreach, New York, v. 4. n. 1, p. 145-152, 2011.

GONZÁLEZ GALLI, L.; REVEL CHION, A.; MEINARDI, E. Actividades centradas en obstáculos para enseñar el modelo de evolución por selección natural. Revista de Educación en Biología, Córdoba, v. 11, n. 1, p. 52-55, 2008.

GREENE, E. The logic of university students' misunderstandings of natural selection. Journal of Research in Science Teaching, Hoboken, v. 27, n. 9, p. 875-885, 1990.

GRIFFITH, J. A.; BREM, S. K. Teaching evolutionary biology: pressures, stress, and coping. Journal of Research in Science Teaching, Hoboken, v. 41, n. 8, p. 791-809, 2004.

HALLDEN, O. The evolution of the species: pupil perspectives and school perspectives. International journal of Science Education, Abingdon, v. 10, n. 5, p. 541-552, 1988. HERNÁNDEZ SAMPIERI, R.; FERNÁNDEZ COLLADO, C.; BAPTISTA LUCIO. M. Metodología de la investigación. 5. ed. México: McGraw-Hill. 2010.

INAGAKI, K.; HATANO, G. Conceptual change in naïve biology. In: VOSNIADOU, S. (Ed.). International handbook of research in conceptual change. Nueva York: Routledge, 2010. p. 263-294.

JIMÉNEZ ALEIXANDRE, M. Cambiando las ideas sobre el cambio biológico. Enseñanza de las Ciencias, Barcelona, v. 9, n. 3, p. 248-256, 1991.

JIMÉNEZ ALEIXANDRE, M. La enseñanza y el aprendizaje de la biología. In: (Coord.). Enseñar ciencias. Barcelona: Grao, 2009.

JUNGWIRTH, E. The problem of teleology in biology as a problem of biology-teacher education. Journal of Biological Education, Philadelphia, v. 9, n. 6, p. 243-246, 1975.

KAMPOURAKIS, K.; ZOGZA, V. Students' intuitive explanations of the causes of homologies and adaptations. Science \& Education, Dordretch, v. 17, n. 1, p. 27-47, 2008.

KAMPOURAKIS, K.; ZOGZA, V. Students'preconceptions about evolution: how accurate is the characterization as "Lamarckian" when considering the history of evolutionary thought? Science \& Education, Dordrecht, v. 16, n. 3-5, p. 393-422, 2006. 
Galli, L. G.; Meinardi, E.

KEIL, F. El nacimiento y enriquecimiento de conceptos por dominios: el origen de los conceptos de seres vivientes. In: HIRSCHFELD, L.; GELMAN, S. (Comp.). Cartografía de la mente: la especificidad de dominio en la cognición y en la cultura. Barcelona: Gedisa, 2002. v. 1, p. 329-357.

KELEMEN, D. Function, goals and intention: children's teleological reasoning about objects. Trends in Cognitive Sciences, Oxford, v. 3, n. 12, p. 461-468, 1999.

KELEMEN, D.; ROSSET, E. The human function compuction: teleological explanation in adults. Cognition, Amsterdam, v. 111, n. 1, p. 138-143, 2009.

KEOWN, D. Teaching evolution: improved approaches for unprepared students. American Biology Teacher, Oakland, v. 50, n. 7, p. 407-410, 1988.

LATORRE BELTRÁN, A.; RINCÓN IGEA, D.; ARNAL, A. Bases metodológicas de la investigación educativa. Barcelona: Hurtado, 1997.

LAWSON, A. E.; THOMPSON, L. D. Formal reasoning ability and misconceptions concerning genetics and natural selection. Journal of Research in Science Teaching, Hoboken, v. 25, n. 9, p. 733-746, 1988.

LEHRER, R.; SCHAUBLE, L. Scientific thinking and science literacy: supporting development in learning in contexts. In: DAMON, W. et al. (Ed.). Handbook of child psychology. 6th. ed. Hoboken: John Wiley, 2006. v. 4, p. 153-196.

LENNOX, J. Darwin was a teleologist. Biology and Philosophy, Dordrecht, v. 8, n. 4, p. 409-421, 1993.

MARTIN, M. An examination of students misconceptions in genetics. In: HELM, H.; NOVAK, J. D. (Ed.). Proceedings of the International Seminar on Misconceptions in Science and Mathematics. Ithaca: Cornell University, 1983. p. 218-225.

MARTINS, C. M. C.; BRAGA, S. A. M. As explicações dos estudantes sobre o processo de adaptação dos seres vivos. In: ENCONTRO PERSPECTIVAS DO ENSINO DE BIOLOGIA, 8., 2002, São Paulo. Anais... São Paulo: USP, 2002. 1 CD-ROM.

MEINARDI, E.; ADÚRIZ-BRAVO, A. Encuesta sobre la vigencia del pensamiento vitalista en los profesores de ciencias naturales. Revista Iberoamericana de Educación, Madrid, 2002. Disponible en: <http://www.rieoei.org/experiencias28.htm>. Acceso el: 08 oct. 2014.

MILLÁN BENÍTEZ, P.; CARMONA PIÑA, R.; ZÁRATE, B. Comprensión y malentendidos del concepto de selección natural en estudiantes universitarios. Revista Mexicana de Investigación Educativa, México, v. 2, n. 3, p. 45-66, 1997.

NEHM, R.; REILLY, L. Biology majors' knowledge and misconceptions of natural selection. BioScience, Washington, v. 57, n. 3, p. 263-272, 2007.

NEHM, R. H.; SCHONFELD, I. S. Does increasing biology teacher knowledge of evolution and the nature of science lead to greater preference for the teaching of evolution in schools? Journal of Science Teacher Education, Dordretch, v. 18, n. 5, p. 699-723, 2007. 
NERSESSIAN, N. Constructing and instructing: the role of "abstraction techniques" in creating and learning physics. In: DUSCHL, R.; HAMILTON, R. (Ed.). Philosophy of science, cognitive science, and educational theory and practice. Albany: Suny Press, 1992. p. 48-68.

OSBORNE, R.; FREYBERG, P. El aprendizaje de las ciencias: influencia de las "ideas previas" de los alumnos. Madrid: Narcea, 1995.

OSIF, B. A. Evolution and religious beliefs: a survey of Pennsylvania high school teachers. American Biology Teacher, Oakland, v. 59, n. 9, p. 552-556, 1997.

PASSMORE, C.; STEWART, J. A modeling approach to teaching evolutionary biology in high schools. Journal of Research in Science Teaching, Hoboken, v. 39, n. 3, p. 185-204, 2002.

PETERFALVI, B. Identificación de los obstáculos por parte de los alumnos. In: CAMILLONI, A. R. W. (Comp.). Los obstáculos epistemológicos en la enseñanza. Barcelona: Gedisa, 2001. p. 127-168.

POZO, J. Más allá del cambio conceptual: el aprendizaje de la ciencia como cambio representacional. Enseñanza de las Ciencias, Barcelona, v. 17, n. 3, p. 513-520, 1999.

POZO, J.; GÓMEZ CRESPO, M. Aprender y enseñar ciencia. 4. ed. Madrid: Morata, 2004.

PRINOU, L.; HALKIA, L.; SKORDOULIS, G. What conceptions do Greek school students form about biological evolution? Evolution: education and outreach, New York, v. 1, n. 3, p. 312-317, 2008.

RIDLEY, M. Evolution. 3. ed. Malden: Blackwell, 2004.

ROSA, V. L. et al. O tema evolução entre professores de biologia não-licenciados: dificuldades e perspectivas. In: ENCONTRO PERSPECTIVAS DO ENSINO DE BIOLOGIA, 8., 2002, São Paulo. Coletânea... São Paulo: Faculdade de Educação da Universidade de São Paulo, 2002. 1 CD-ROM.

RUSE, M. Teleology: yesterday, today, and tomorrow? Studies in History and Philosophy of Science Part C: Studies in History and Philosophy of Biological and Biomedical Sciences, Kidlington, v. 31, n. 1, p. 213-232, 2000.

SCOTT, P.; ASOKO, H.; LEACH, J. Students conceptions and conceptual learning in science. In: ABELL, S.; LEDERMAN, N. Handbook of research on science education. New Jersey: Lawrence Elrbaum, 2007. p. 31-56.

SETTLAGE, J. Conceptions of natural selection: a snapshot of the sense-making process. Journal of Research in Science Teaching, Hoboken, v. 31, n. 5, p. 449-457, 1994.

SINATRA, G.; BREM, S.; EVANS, M. Changing minds?: implications of conceptual change for teaching and learning about biological evolution. Evolution: education and outreach, New York, v. 1, n. 2, p. 189-195, 2008. 
Galli, L. G.; Meinardi, E.

SMITH, M. Current status of research in teaching and learning evolution. I. philosophical/ epistemological issues. Science \& Education, Dordrecht, v. 19, n. 4-8, p. 523-538, 2010a.

Current status of research in teaching and learning evolution: II. pedagogical issues.

Science \& Education, Dordrecht, v. 19, n. 4-8, p. 523-538, 2010 b.

SOUTHERLAND, S. A. et al. Understanding students' explanations of biological phenomena: conceptual frameworks o p-prims? Science Education, Hoboken, v. 85, n. 4, p. 328-348, 2001.

STERELNY, K.; GRIFFITHS, P. Sex and death: an introduction to philosophy of biology. Chicago: The University Chicago Press, 1999.

TALANQUER, V. Commonsense chemistry: a model for understanding students' alternative conceptions. Journal of Chemical Education, Washington, v. 83, n. 5, p. 811-816, 2006.

THOMAS, G. Metacognition in science education: past, present and future considerations. In: FRASER, B. J.; TOBIN, K.; McROBBIE, C. J. (Ed.). Second international handbook of science education. London: Springer, 2012.

THOMPSON, P. The structure of biological theories. Albany: State University of New York Press, 1989.

VEENMAN, M. Metacognition in science education: definitions, constituents, and their intricate relation with cognition. In: ZOHAR, A.; DORI, Y. J. (Ed.). Metacognition in science education: trends in current research. London: Springer, 2012. p. 21-36.

VOSNIADOU, S. Investigaciones sobre el cambio conceptual: direcciones futuras y de vanguardia. In: SCHNOTZ, W.; VOSNIADOU, S.; CARRETERO, M. (Comp.). Cambio conceptual y educación. Buenos Aires: Aique, 2006. p. 35-52.

Reframing the classical approach to conceptual change: preconceptions, misconceptions and synthetic models. In: FRASER, B.; TOBIN, K.; McROOBBIE, C. (Ed.). Second international handbook of science education. London: Springer, 2012. p. $119-130$.

ZOHAR, A.; DORI, Y. J. (Ed.). Metacognition in science education: trends in current research. London: Springer, 2012.

ZOHAR, A.; GINOSSAR, S. Lifting the taboo regarding teleology and anthropomorphism in biology: education-heretical suggestions. Science Education, Hoboken, v. 82, n. 6, p. 679-697, 1998.

Artigo recebido em 07/11/13. Aceito em 24/07/14. 